\title{
Axitinib destined for second place?
}

Two trials recently published in

The Lancet Oncology demonstrate the clinical activity of axitinib in treatment-naive patients; however, disappointing survival outcomes suggest it will remain a second-line treatment option for metastatic renal cell carcinoma (mRCC) for the time being.

The first trial, reported by Thomas Hutson and colleagues, provides the first phase III data of first-line axitinib treatment in patients with mRCC. Encouraged by the positive findings of the AXIS trial-which tested axitinib against sorafenib in the secondline setting-Hutson and his team amended their similar ongoing trial of axitinib versus sorafenib to include a cohort of treatment-naive patients with mRCC.

Patients, predominantly from Eastern Europe, were randomized to receive either axitinib $5 \mathrm{mg}$ twice daily $(n=189)$ or sorafenib $400 \mathrm{mg}$ twice daily $(n=96)$ in 4-week cycles. Median progression-free survival (PFS) the primary end point-was higher for axitinib than sorafenib (10.1 months versus 6.5 months) but unfortunately did not reach statistical significance. The investigators note that their predicted improvement in PFS for axitinib (4.3 months) was ambitious and, therefore, their study was underpowered.

In addition, they theorize that the non-US patient population might explain the discrepancy between this trial and AXIS. Local practice patterns are likely to affect the type of patient enrolled and how they are managed. Geographical considerations also influenced the choice of comparator in this trial, leading to selection of sorafenib rather than a standard first-line option, such as sunitinib or pazopanib.

On the plus side, however, significantly more patients who received axitinib achieved an objective response (assessed by an independent review committee)

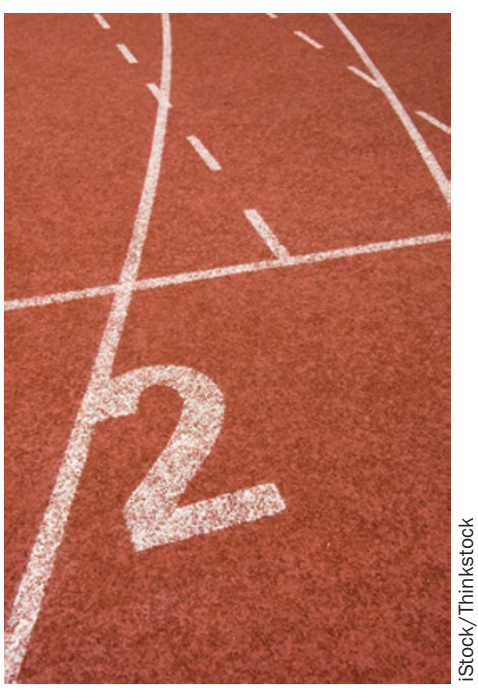

than those who took sorafenib, which confirms the clinical activity of axitinib in this context.

Clinical activity in the first-line setting was also demonstrated in the second study, a phase II trial in which Brian Rini and his team assessed the use of axitinib titration in treatmentnaive patients. They found that more patients who underwent axitinib dose titration achieved an objective response than those who received placebo titration, supporting the concept of this treatment regimen.

Investigators are clearly working hard to elucidate the optimal use of axitinib; however, as Nadia Yousaf and James Larkin point out in their comment that accompanies the phase III trial, perhaps efforts would be better focused on the identification and development of molecular predictive biomarkers for mRCC, which are currently sorely lacking.

\section{Sarah Payton}

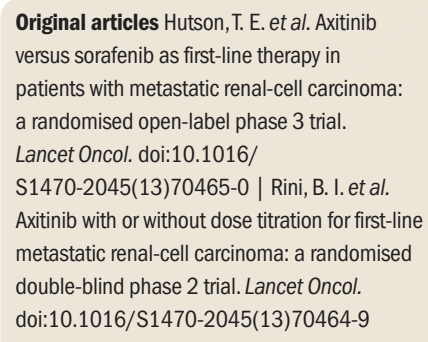

